\title{
Silicon-based micro direct methanol fuel cell with an N-inputs-N-outputs anode flow pattern
}

\author{
ZHANG YuFeng ${ }^{1,2^{*}}$, WANG LuWen ${ }^{1}$, YUAN Zhen Yu ${ }^{1}$, WANG ShiBo ${ }^{1}$, LI JianMin ${ }^{1} \&$ \\ LIU XiaoWei ${ }^{1,2}$ \\ ${ }^{1}$ MEMS Center, Harbin Institute of Technology, Harbin 150001, China; \\ ${ }^{2}$ Key Laboratory of Micro-Systems and Micro-Structures Manufacturing, Ministry of Education, Harbin 150001, China
}

Received August 4, 2010; accepted November 5, 2010

\begin{abstract}
A micro direct methanol fuel cell ( $\mu \mathrm{DMFC})$ is suitable for use in notebook computers, mobile phones, and other digital products. To resolve the poor mass-transport efficiency problem in the anode flow channel, this paper presents an $\mathrm{N}$-inputs-N-outputs parallel flow pattern with rectangular convexes to reinforce methanol mass transport and reduce concentration polarization. The simulation results show that the $\mathrm{N}$-inputs-N-outputs parallel flow channels with the rectangle convexes improve the performance. $\mu$ DMFCs, which have four anode flow patterns, are fabricated using MEMS (microelectromechanical systems) technology. The experimental results show that the $\mu$ DMFC with the rectangle convexes has a performance better than previously reported systems, and has a peak power density of $19.96 \mathrm{~mW} / \mathrm{cm}^{2}$. The simulation and experimental results are in good agreement.
\end{abstract}

micro direct methanol fuel cell, MEMS, NINO, convexes

Citation: Zhang Y F, Wang L W, Yuan Z Y, et al. Silicon-based micro direct methanol fuel cell with an N-inputs-N-outputs anode flow pattern. Chinese Sci Bull, 2011, 56: 826-829, doi: 10.1007/s11434-010-4335-5

Micro direct methanol fuel cells ( $\mu$ DMFC) possess many advantages, such as high energy conversion, fuel efficiency, room temperature operation, simple structure, and high power density. They are based on MEMS (microelectromechanical systems) technology. In addition, they have reached a breakthrough point and will likely be used in micro systems and portable electronic products such as notebook computers and mobile phones in the near future [1-5]. Because of the potential of $\mu \mathrm{DMFC}$ technology to replace conventional power sources, many researchers have paid increased attention to various aspects concerning the $\mu \mathrm{DMFC}$ technology [6-9], such as the anode-flow-field configuration, which is a crucial factor for $\mu \mathrm{DMFC}$ applications $[10,11]$.

Structurally, a $\mu \mathrm{DMFC}$ consists of two current collectors (anode and cathode) with flow fields and a membrane electrode assembly (MEA) between them. In $\mu$ DMFCs, an aqueous

\footnotetext{
*Corresponding author (email: yufeng_zhang@hit.edu.cn)
}

methanol solution and oxygen are fed into the anode and cathode, respectively. Through electrochemical reactions with the water, the methanol is oxidized and produces electrons, protons, and carbon dioxide. The electrons produced at the anode carry the free energy charge of the chemical reaction and perform electrical work on the external load. In addition, the protons can transport through a proton exchange membrane to the cathode, where the electrons come back from the external circuit to form water molecules with the protons. Through the oxidation and deoxidization processes of the electrochemistry reactions, the electricity is exported continuously.

The current collector is a vital component of the $\mu \mathrm{DMFC}$ [12], which not only supplies a passage for the transport of reactants, but also provides structural support for the weak MEA and collects current. The design of the anode flow field is critical to achieving optimized performance in the $\mu \mathrm{DMFC}$. And many flow field structures have been reported [13-15], such as parallel channel, serpentine channel, 
and spiral channel. The parallel flow field structure, which has an easy to fabricate simple structure and small channel drag, can generally be used as the anode current collector of a $\mu \mathrm{DMFC}$, especially applied as the stack architecture. However, a $\mu$ DMFC with a parallel anode structure has a lower fuel utilization efficiency than other structures because of the outflow of a mass of methanol from the outlet, and the methanol cannot be supplied effectively for high-current operation [10].

To address these disadvantages, this paper proposes an $\mathrm{N}$-inputs-N-outputs (NINO) parallel flow field structure for the anode current collector of the $\mu \mathrm{DMFC}$. This is shown in Figure 1. It can be seen that the number of inputs and outputs for the NINO structure is the same as that of the channels in the parallel flow field. In addition, every channel possesses an input and an output, and this guarantees the unification of the methanol concentration at the inputs with the well-distributed methanol in every parallel channel. Therefore, the performance of collecting current can be improved, and the reaction product, $\mathrm{CO}_{2}$, can be removed immediately. Because of the independent channels, the cell will not stop operating even if one channel is obstructed. However, in the NINO parallel flow field structure each single channel is relatively short and has a lower solution-pressure gradient between the input and the output, hinders methanol mass transport in the flow direction. To improve the cell performance by reinforcing the anode methanol mass transport, this work presents three modified parallel flow fields based on the above NINO structure. In these three proposed structures, triangular, rectangular and semicircular convexes are embedded in every channel in the NINO parallel flow field. This is shown in Figure 2.

\section{Design}

To demonstrate the improvement of the channel structure proposed in this paper and identify the optimal flow pattern, we simulated the four flow patterns of the NINO structure, which are shown in Figure 2. In the model descriptions, the convection-diffusion equation was used to characterize the methanol

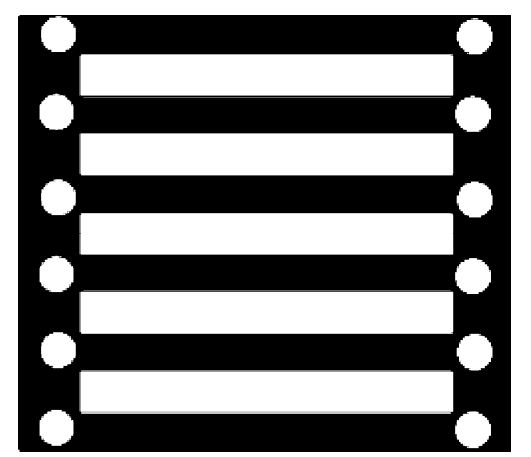

Figure 1 Schematic of the anode NINO parallel flow field structure.

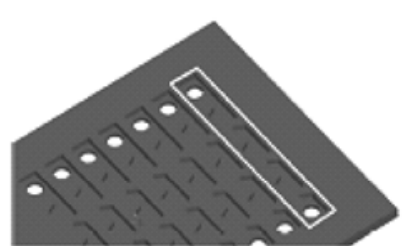

(a)
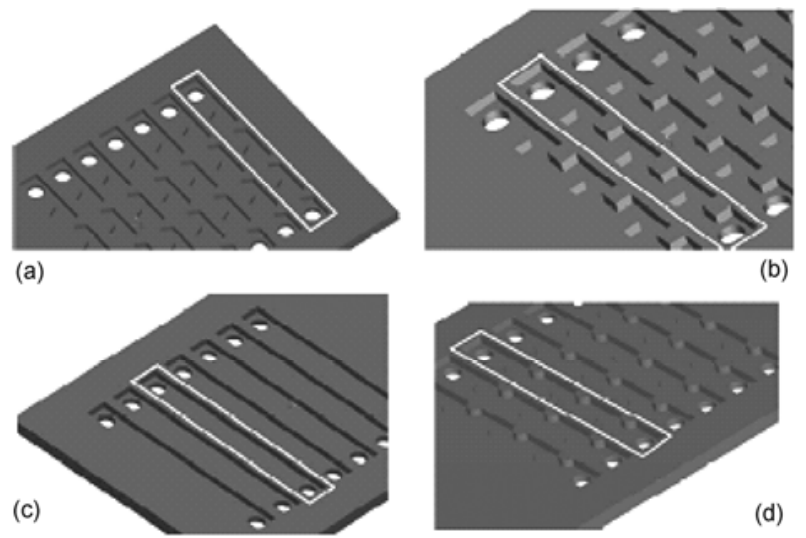

(b)

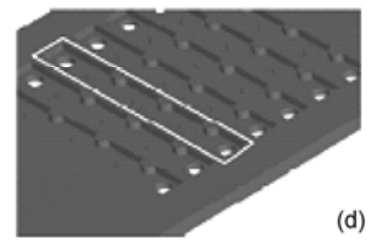

(d)
Figure 2 Perspective of the four NINO parallel flow patterns. (a) NINO flow pattern with triangular convexes; (b) NINO flow pattern with rectangular convexes; (c) NINO pattern without convexes; (d) NINO flow pattern with semicircular convexes.

mass distribution in the flow channel and diffusion layer.

$$
\nabla \cdot\left(-D_{\text {eff }} \nabla c\right)=R-\boldsymbol{u} \nabla c,
$$

where $D_{\text {eff }}$ is the effective diffusion coefficient of the methanol in the diffusion layer; $c$ denotes the methanol concentration; $R$ denotes the reaction rate of the methanol; $\boldsymbol{u}$ is the velocity vector of the liquid phase.

Considering that the methanol solution flow in the channel can be classified as a laminar flow, the incompressible Navier-Stokes equations can be applied to describe the momentum transport in the flow field.

$$
\begin{gathered}
\rho_{l} \boldsymbol{u} \cdot \nabla \boldsymbol{u}=\nabla\left[-p+\eta\left(\nabla \boldsymbol{u}+(\nabla \boldsymbol{u})^{\mathrm{T}}\right)\right]+F, \\
\nabla \cdot \boldsymbol{u}=0,
\end{gathered}
$$

where $\rho_{l}$ is the density of the methanol solution; $p$ denotes the pressure of the liquid phase; $\eta$ denotes the dynamic viscosity; $F$ is the volume force.

Moreover, the mass-momentum transport in a porous medium is characterized by the Darcy law, because the diffusion layer (carbon cloth or carbon paper) is porous.

$$
\boldsymbol{u}=-\frac{\kappa}{\eta} \nabla p
$$

where $\kappa$ is the permeability of the liquid phase.

After coupling the above equations, the numerical analysis was carried out using the COMSOL Multiphysics software (Massachusetts, USA), and the simulation results are shown in Figure 3. Figure 3(a) shows the pressure distribution of the four flow patterns units of the NINO structure. Comparing the four flow patterns of the NINO structure, it was found that the channels with convexes had a higher pressure difference than the smooth channel without convexes, and the flow pattern with the square convexes has the highest pressure difference. Figure 3(b) shows the flow-rate distributions at the anode interfaces between the flow fields 

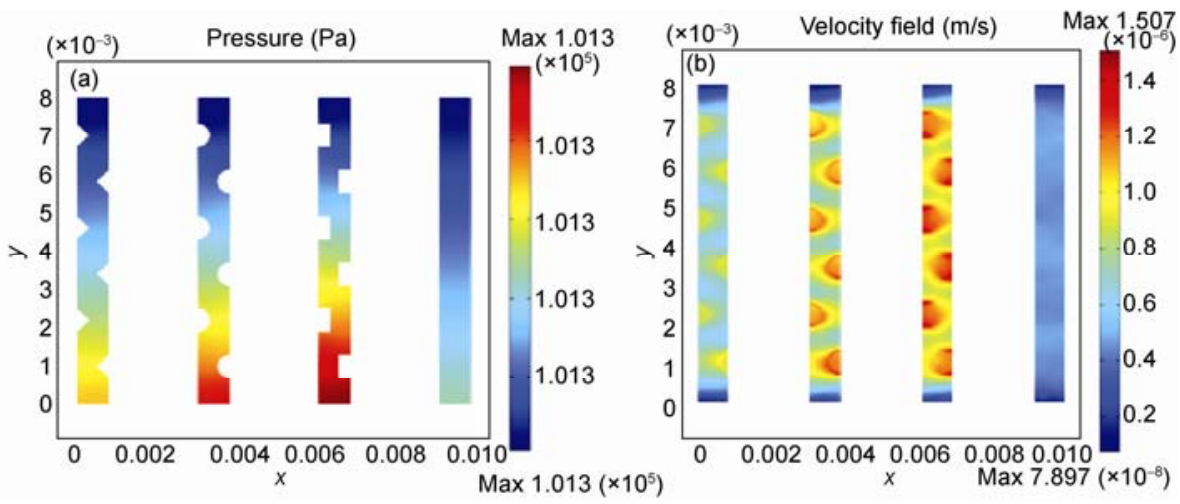

Figure 3 Pressure and flow-rate distributions of the four flow patterns of the NINO structures (from left to right: triangular, semicircular, rectangular, and without convexes). (a) The pressure distributions of the four flow patterns; (b) the flow-rate distributions of the four flow patterns.

and the diffusion layers. It can be seen that the flow-rate distribution in the flow channels with convexes are more uneven than for the channel without convexes. However, the NINO parallel flow field with rectangular convexes has an advantage over the other systems with convexes in its flow-rate distribution. Because the convexes in the flow channel contribute to the local laminar flow in the methanol solution flow process, a more concentrated distribution of solution will result. Therefore, the methanol mass transport in the diffusion layer can be strengthened and the concentration polarization of the $\mu \mathrm{DMFC}$ can be reduced, which will improve the performance.

\section{Fabrication and results}

To verify the simulation results, the current collectors with four different flow patterns were fabricated using siliconbased MEMS technologies, including CVD, photolith- ography, etching, and sputtering. DuPont Nafion 117 (Delaware, USA) was used as the proton exchange membrane of the $\mu$ DMFCs. The catalyst for the anode was coated with the noble metal, Pt-Ru/C, and the loading level of Pt in the anode-catalyst layer was $4.0 \mathrm{mg} / \mathrm{cm}^{2}$. The catalyst for the cathode was carbon-supported $\mathrm{Pt}$, and loading level of $\mathrm{Pt}$ in the cathode catalyst layer was also $4.0 \mathrm{mg} / \mathrm{cm}^{2}$. Carbon paper was used as the diffusion layer in the $\mu$-DMFC. These parts were then assembled to form the Membrane Electrode Assembly (MEA). Ultimately, the MEA was sandwiched between the anode and cathode to create an integrated $\mu$ DMFC with an active area of $0.64 \mathrm{~cm}^{2}$. Figure 4 shows a photograph of the $\mu$ DMFC prototype structure fabricated using micro-machining technology.

Performance tests of the $\mu$ DMFCs with four flow patterns were performed using a $1.5 \mathrm{~mol} / \mathrm{L}$ methanol solution at $0.5 \mathrm{~mL} / \mathrm{min}$ at room temperature. The results are plotted in Figure 5. The NINO parallel flow pattern with no convexes was used as the cathode flow structure. Oxygen was supplied to the cathode at a constant gas flow rate of 50

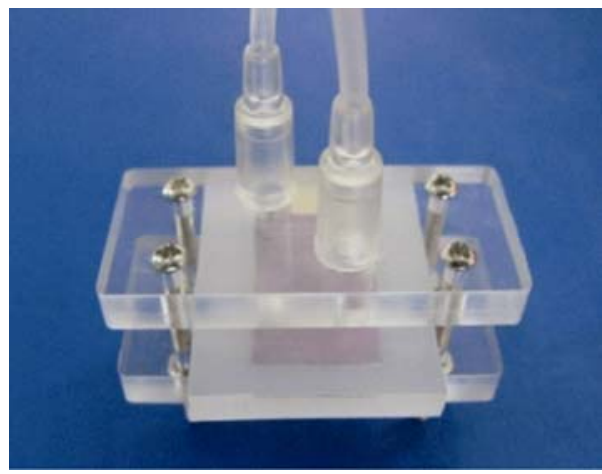

Figure 4 Photograph of an assembled $\mu$ DMFC.

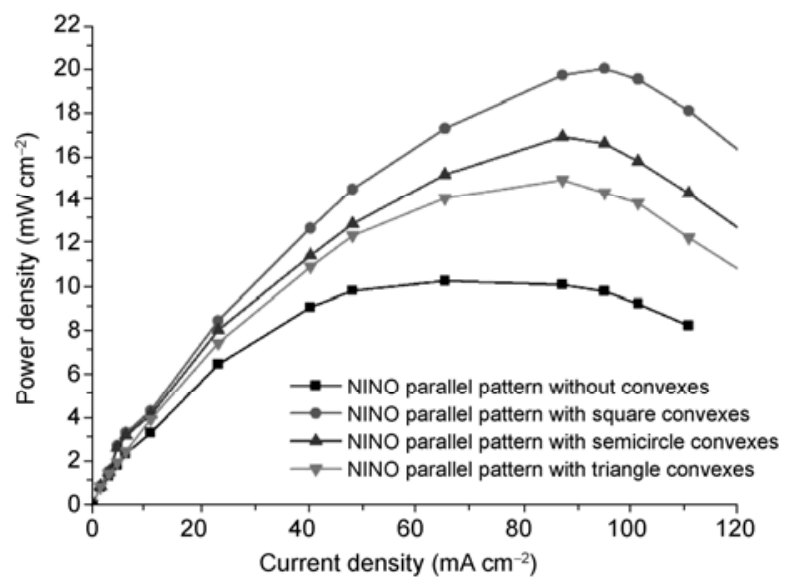

Figure 5 Performance comparison of the $\mu$ DMFCs with the four different NINO parallel flow patterns at room temperature.

$\mathrm{mL} / \mathrm{min}$. From the testing results, it can be seen that the order of voltage performance from highest to lowest for the NINO parallel flow fields was rectangular, semicircular, triangular, and without convexes for a given current density. It can also be seen that the power density of the $\mu \mathrm{DMFC}$ with rectangular convexes performed the best with a maximum power density of $19.96 \mathrm{~mW} / \mathrm{cm}^{2}$ at a current density of 95 $\mathrm{mA} / \mathrm{cm}^{2}$. The tests agreed with the simulation results. This 
shows that a NINO anode parallel flow field design with rectangular convexes can effectively improve methanol mass transport and, consequently, reduce the concentration polarization. When the requirements of supply-voltage applications are considered, this NINO parallel flow pattern design with rectangular convexes is more appropriate for the realization of a stack architecture, which unlike conventional flow-field structures can be applied in portable systems.

\section{Conclusions}

In conclusion, a silicon-based $\mu \mathrm{DMFO}$ with NINO anode flow patterns was presented. Compared with other NINO flow fields with different convexes, the $\mu \mathrm{DMFC}$ anode with rectangular convexes was able to reinforce methanol mass transport and reduce concentration polarization utilizing the simulation analysis. The $\mu$ DMFCs were fabricated using silicon-based MEMS technology. Due to the increase in methanol mass transport efficiency from the channel to the electrode, the $\mu \mathrm{DMFC}$ with the rectangular-convex NINO flow field yielded a maximum power density of 19.96 $\mathrm{mW} / \mathrm{cm}^{2}$ at room temperature. From the results obtained in this work, it can be concluded that the anode NINO flow field demonstrated in the $\mu \mathrm{DMFC}$ is also applicable to the greater DMFCs running on the anode.

This work was supported by the National Natural Science Foundation of China (60806037 and 61076105), the National High-Tech Research \& Development Program of China (2006AA04Z353), the Natural Science Research Innovation Foundation of the Harbin Institute of Technology (HIT. NSRIF. 2009008) and the Key Laboratory Opening Funding of Key Laboratory of Micro-Systems and Micro-Structures Manufacturing of the
Ministry of Education (HIT. KLOF. 2009003).

1 Kamarudin S K, Achmad F, Daud W R W. Overview on the application of direct methanol fuel cell (DMFC) for portable electronic devices. J Hydrogen Energy, 2009, 34: 6902-6916

2 Pichonat T, Gauthier-Manuel B. Recent developments in MEMSbased miniature fuel cells. Microsyst Technol, 2007, 13: 1671-1678

3 Kundu A, Jiang J H, Gil J H, et al. Micro-fuel cells-Current development and applications. J Power Sources, 2007, 170: 67-78

4 Kamarudin S K, Daud W R W, Ho S L, et al. Overview on the challenges and developments of micro-direct methanol fuel cells (DMFC). J Power Sources, 2007, 163: 743-754

5 Li C N, Sun G Q, Ren S Z, et al. Modification of perfluorosulfonic acid membrane applied in DMFC. Chinese Sci Bull, 2005, 50: 2049-2054

6 Lu G Q, Wang C Y. Development of micro direct methanol fuel cells for high power applications. J Power Sources, 2005, 144: 141-145

7 Dai H, Zhang H M, Luo Q T, et al. Properties and fuel cell performance of proton exchange membranes prepared from disulfonated poly (sulfide sulfone). J Power Sources, 2008, 185: 19-25

8 Zhang Y F, Zhang P, Zhang B, et al. Development of an air-breathing direct methanol fuel cell with the cathode shutter current collectors. J Hydrogen Energy, 2010, 35: 5638-5646

9 Zhang B, Zhang Y F, He H, et al. Development and performance analysis of a metallic micro direct methanol fuel cell for high-performance applications. J Power Sources, 2010, 195: 7338-7348

10 Zhang Q, Wang X H, Zhong L Y, et al. Design, optimization and microfabrication of a micro-direct methanol fuel cell with microblocks in anode structure. Sens Actuators A: Physical, 2009, 154: 247-254

11 Wong $\mathrm{C} \mathrm{W}$, Zhao T S, Ye Q, et al. Experimental investigations of the anode flow field of a micro direct methanol fuel cell. J Power Sources, 2006, 155: 291-296

12 Qian P, Zhang H M, Chen J, et al. A novel electrode-bipolar plate assembly for vanadium redox flow battery applications. J Power Sources, 2008, 175: 613-620

13 Yang H, Zhao T S. Effect of anode flow field design on the performance of liquid feed direct methanol fuel cells. Electrochim Acta, 2005, 50: 3243-3252

$14 \mathrm{Xu} \mathrm{C}$, Zhao T S. A new flow field design for polymer electrolyte-based fuel cells. Electrochem Commun, 2007, 9: 497-503

15 Oliveira V B, Rangel C M, Pinto A M F R. Effect of anode and cathode flow field design on the performance of a direct methanol fuel cell. J Chem Eng, 2010, 157: 174-180

Open Access This article is distributed under the terms of the Creative Commons Attribution License which permits any use, distribution, and reproduction in any medium, provided the original author(s) and source are credited. 